\title{
Spline Based Trajectory Planning for Cooperative Crane Lifts
}

\author{
S. Vijaya Bhaskar, N. Ramesh Babu, Koshy Varghese \\ Indian Institute of Technology Madras \\ Chennai 600036, India \\ nrbabu@iitm.ac.in
}

\begin{abstract}
This paper reports the work done towards planning the trajectory for cooperative crane lifts. The location of the hook points between the pick and place positions are determined using a path-planner. These are inputs to the trajectory planner. The goal of the trajectory planner is to connect all the path-points while ensuring the overshoots, velocities, accelerations and jerk for each degree of freedom to be within acceptable limits for the system. A number of alternate techniques are available for defining the trajectory between the path-points. This paper reports on the evaluation of algebraic and trigonometric splines for trajectory planning. Splines of various orders were initially evaluated for a single joint. This evaluation found that $4^{\text {th }}$ order trigonometric splines were most appropriate. Hence, this was applied to a lift problem involving the $2 \times 4$ DOF cooperative manipulator system and the results are presented.
\end{abstract}

Key Words: Trajectory planning, Trignometric spline, Cooperative manipulators, Heavy lift planning

\subsection{INTRODUCTION}

Motion planning is an important area of research in robotics. One of the goals of motion planning is to develop autonomous systems to facilitate manipulators to maneuver in an unstructured or unknown environment. Without motion planning algorithms, the motions of a manipulator need to be constantly specified by the operator.

In real-time, an autonomous motion planner complimented by appropriate sensors, will enable a manipulator system to plan its own course of action on-line without human intervention. If the environment is highly structured, motion planning can also be done off-line.

Based on the specification of the task, motion planning entails either planning a trajectory along a specified path or planning both a path and a trajectory. While a path is specified by points in Cartesian space (or in joint space) to describe the spatial evolution of the end-effector (or the joints), a trajectory is a curve in state space that describes the system evolution in time [1].

Robot motions are specified in two different ways. When the end-effector is constrained to a predetermined path, the motion is called path-constrained. Applications of pathconstrained manipulation include welding, gluing and spraying. Since the path is imposed by the task, only trajectory planning is addressed. When only the initial and final positions (and velocities) of the end-effector are imposed, the motion is called point-to-point. Pick and place operations are point-to-point manipulations. In this case, both path and trajectory must be determined.

Among several application areas of robots, material handling is one area where motion planning of single/multiple manipulators is required for moving the end effector from point-to-point. In some situations, heavy long objects such as pressure vessels, boiler, process columns, chimneys etc. that can weigh between 100 tons to
1000 tons are handled by large capacity manipulators. As these manipulators pose certain difficulties such as their limited availability, difficulty in transportation and high utilization cost, there is a potential to replace them by medium capacity manipulators, which can cooperatively handle such heavy long objects.

This paper presents the work done towards motion planning of cooperative crane-like manipulators. The path between the pick and place points is determined using a Genetic Algorithm as described in [2]. The task of the motion planner is to determine a suitable trajectory passing through these points using interpolation techniques. In addition to passing through the defined path-points, the motion planner should ensure the acceptability of velocity profile as well as the acceleration and jerk experienced by the system for the lifting application. There are a number of interpolation techniques available and the suitability of these techniques for the cooperative crane problem need to be studied initially.

Thus specific objectives of the present research work are:

- To investigate the suitability of different interpolation techniques for trajectory planning of multiple robots

- To apply the technique selected to generate the profiles for different joints of $2 \times 4$ cooperative robots while executing the trajectory.

The scope of the work is limited to :

- Two identical manipulators, each with 4 degrees of freedom

- The base of each robot is fixed during the operation

- All obstacles in the work space are assumed to be static

- Dynamics for the prototype model is considered

The methodology utilized investigates the applicability of algebraic splines, and trigonometric splines to a single 
DOF system initially. Based on the results, the best technique is applied to arrive at the profiles for cooperative crane type manipulator system.

\subsection{PAST WORK}

Motion planning includes three basic activities such as sensing, planning and actuation. Trajectory planning is a subset of motion planning which refers to planning of collision free optimal path with time. Path planning is a subset of trajectory planning which focuses on the planning of path without time. A number of researchers are involved in developing the trajectory planning techniques for robots. However, only few research groups have considered trajectory planning of robots with obstacles in workspace. Some activities in this direction are discussed in this section.

\subsection{Trajectory planning of a single robot}

Several attempts were made towards trajectory planning for a single robot. Attempts to plan the trajectory of a single robot using polynomial techniques were addressed [3][4]. Recent review on trajectory planning for single robots has addressed the application of interpolation techniques like trigonometric splines, B-splines. An efficient trajectory technique based on combination of algebraic and trigonometric splines, which is very useful in obstacle avoidance, was developed [5]. A technique that is useful for online-planning or moving obstacle cases, using B-splines, was developed [6].

A method to formulate the trigonometric splines for trajectory planning of robots was developed [7]. They gave the examples for one link and one set of angles with different time intervals. Neural networks to optimize the velocity, acceleration values of cubic splines in order to get the minimum jerk trajectories, was utilised [8].

The optimal trajectory generation between two points using cubic polynomial with genetic algorithms was generated [9]. Simulated results were reported for the above methods considering two-link planar manipulator. A method to generate optimal trajectory considering moving obstacles in workspace was presented [10]. Two-link planar manipulator was considered for simulation purpose by considering the optimal traveling time and minimum mechanical energy of the actuators.

An efficient method for trajectory planning using GA was developed [11]. In this work, GA concepts were utilized for choosing the polynomial coefficients to generate the optimal trajectory. A two-link manipulator was considered for generating the trajectories between two points in Cartesian space without considering the intermediate configurations.

\subsection{Trajectory planning of cooperative robots}

The trajectory planning for cooperative robots is difficult due to the complexity in formulation of system dynamics. A method for collision free trajectory planning for cooperative multiple manipulators is presented [12]. In this work, the system dynamics were expressed by path parameters and trajectory simulation is shown for $2 \times 2$ planar manipulator with obstacles in the workspace.

Effective material handling, involving trajectory planning, was developed for multiple coordinating robots using genetic algorithm considering initial and final configurations without intermediate configurations [13] [14]. As the intermediate configurations were not considered, obstacle avoidance strategies were not modeled. Further, the manipulators considered did not possess linear and flexible links such as the telescoping and the hoist movements of a crane-like manipulator.

\subsection{PROBLEM DEFINITION}

The present system consists of two crane type robots having similar D.O.F like crane as shown in Figure 1. Each robot of present cooperative system has a maximum of four degrees of freedom. In general, the crane robot has eight D.O.F. They are mobility of base in plane i.e. along ' $\mathrm{x}$ ' and ' $\mathrm{y}$ ' directions $[C x, C y]$, swing of base $[\varphi]$, luff of boom $[\theta]$, telescoping action of boom $[\psi]$, hoisting action of rope $[h]$, rotation of hoisting rope about ' $x$ ' and ' $y$ ' axes $\left[h_{x}, h_{y}\right]$. Thus, a unique configuration of crane type robot can be represented by the configuration set $[C x, C y, \varphi, \theta$, $\left.\psi, h, h_{x}, h_{y}\right]$.

The scope of the present work is limited to fixed base type, i.e. the movement of base is not considered. In the view of this, the configuration set of a single robot is further simplified into $\left[\varphi, \theta, \psi, h, h_{x}, h_{y}\right]$. Here, it should be noted that the free swinging or rotation of the object about the hoisting rope due to the wind or inertia of the object is neglected since the object is held by two manipulators through rigging. In case of a single robot, it is necessary to consider the free rotation of the object.

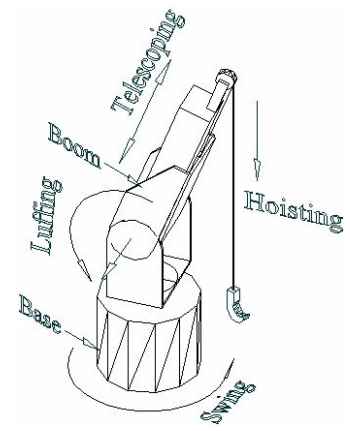

Figure 1 D.O.F of a single robot used in cooperative robot system

A schematic diagram representing the cooperative robots handling an object is shown in Figure 2. The active degrees of freedom for a single manipulator in $2 \times 4$ cooperative systems is given as swing, luff, telescope, and hoist and presented by $[\varphi, \theta, \psi, \mathrm{h}]$. The configuration of $2 \times 4$ cooperative manipulator system i.e. two manipulators with four degrees of freedom, is expressed as $\left\{[\varphi, \theta, \psi, h]_{1}\right.$, 
$[\varphi, \theta, \psi, h]_{2}$. This problem is referred as $2 \times 4$ crane type robot problem, since it involves two robot type manipulators, each with four degrees of freedom.

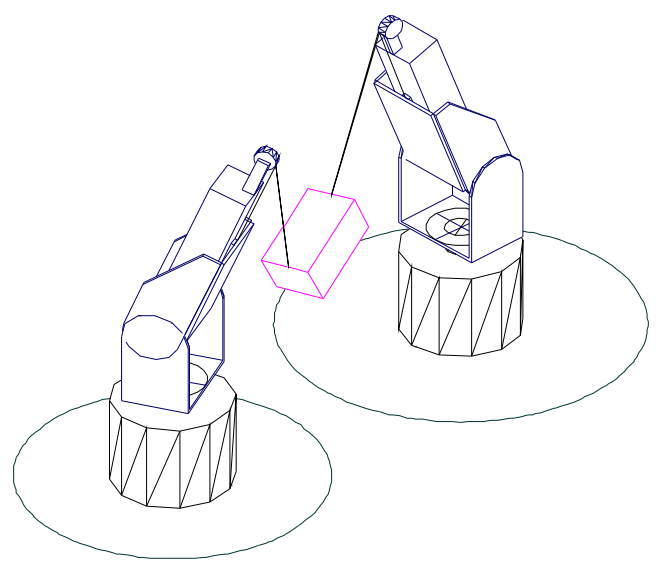

Figure 2. Schematic diagram of cooperative robot system

\subsection{Limits of Movement for Robot}

Each robot supports only a certain range of movement, for different DOF. These limits describe the workspace of the robot system. Table 3.1 shows the limits of movement for different links.

Table 3.1 DOF limits

\begin{tabular}{|c|c|c||}
\hline DOF (Name) & Minimum Limit & Maximum Limit \\
\hline Swing (Base) & -180 & 180 \\
\hline Luff(Boom) & 10 & 80 \\
\hline Telescope & $0 \mathrm{~cm}$ & $15 \mathrm{~cm}$ \\
\hline Hoist & $0 \mathrm{~cm}$ & $\begin{array}{c}\text { Depends on the } \\
\text { luff angle load } \\
\text { geometry }\end{array}$ \\
\hline
\end{tabular}

\subsection{Approach for Trajectory Planning}

The desired path is usually specified in terms of the motion of the end effector of a robot in Cartesian space by a set of points, each of which describes the desired position and orientation of the end effector at that point. Each path point in Cartesian space can therefore be represented in general by six dimensional vector, with three elements being used to specify the position and three to specify the orientation.

However, in this model load orientation is not considered as discussed earlier. Generally, by applying inverse kinematics transformations corresponding to the robot under consideration, the path points in Cartesian space can be converted into a set of path points in joint space. For this work, the path points in joint space are directly obtained from GA based path planning algorithm [2]. The path is specified as a series of joint positions and is shown as

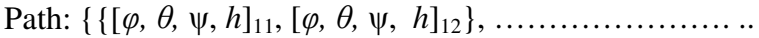
,$\left.\ldots .,\left\{[\varphi, \theta, \psi, h]_{\mathrm{n} 1},[\varphi, \theta, \psi, h]_{\mathrm{n} 2}\right\}\right\}$ $\left\{[\varphi, \theta, \psi, h]_{11},[\varphi, \theta, \psi, h]_{12}\right\}$ represents the angles of swing, luff, telescope, hoist of robot 1 and robot 2 at pick position. $\left\{[\varphi, \theta, \psi, h]_{\mathrm{n} 1},[\varphi, \theta, \psi, h]_{\mathrm{n} 2}\right\}$ represents the respective angles at place position. There are values inbetween the pick and place positions which represent the intermediate configuration angles of various joints of cooperative robots at intermediate path points.

The times at each path point can be chosen arbitrarily or they can be chosen by some heuristic function. Since the present work is not based on optimal time as objective function, the time frame is chosen to suit the capability of the robot systems ability to handle velocity, acceleration and jerk. For each joint, a smooth function in terms of time is determined which passes through all the path points. The function used for a particular joint does not depend on the function used for the other joints. However, it is necessary to ensure that the same time is taken by each joint to complete a segment. This enables the hook to pass through the specified path points in Cartesian space.

\subsection{SPLINE INTERPOLATION TECHNIQUES}

\subsection{Algebraic Splines}

Algebraic splines are widely adopted in the robotics field. These are splines are formed by joining of piecewise algebraic polynomial segments. The exponent of the piecewise polynomial segments determine the order of the spline.

\subsubsection{Cubic and Quintic Splines}

Cubic spline is formulated by joining of piecewise cubic polynomial segments of order 3. Consider the velocity of joint at time $t_{0}$ is $v_{0}$ and $v_{n}$ at time $t_{n}$ and segmental times represented by $h_{i}=t_{i}-t_{i-1}, i=1, \ldots, n$. Then, the cubic polynomial equation representing the position profile of the joint in $i^{\text {th }}$ segment is

$\theta_{i}(t)=a_{i}+b_{i} t+c_{i} t^{2}+d_{i} t^{3} \quad i=1, \ldots, n-1$

where $n$ represents the number of path points or knot points. The number of coefficients i.e. $4(n-1)$ can be determined considering the following initial and final conditions i.e. $i=0$ and $i=n$

At time $t=t_{0}$ and $t=t_{n}$

$$
\left\{\begin{array}{l}
\theta_{1}\left(t_{0}\right)=q_{0} \\
\theta_{n}\left(t_{n}\right)=q_{n}
\end{array}\right.
$$

where $q_{0}$ and $q_{n}$ are the joint angles of a joint at pick and place positions respectively.

$$
\left\{\begin{array}{l}
\dot{\theta}_{1}\left(t_{0}\right)=v_{0} \\
\dot{\theta}_{n}\left(t_{n}\right)=v_{n}
\end{array}\right.
$$


The velocity at initial and final points of the trajectory should be equal to zero.

For any intermediate point: $(i=1, \ldots, n$-1), i.e. for the segment $i$ and $(i+1)$ at time $t=t_{i}$

$$
\theta_{i}\left(t_{i}\right)=\theta_{i+1}\left(t_{i}\right)=q_{i}
$$

where $q_{i}$ is the joint angle at any intermediate point ' $\mathrm{i}$ '. The final velocity and acceleration of first segment is equal to starting velocity and acceleration of next second segment and so on

$$
\left\{\begin{array}{l}
\dot{\theta}_{i}\left(t_{i}\right)=\dot{\theta}_{i+1}\left(t_{i}\right) \\
\ddot{\theta}_{i}\left(t_{i}\right)=\ddot{\theta}_{i+1}\left(t_{i}\right)
\end{array}\right.
$$

By imposing the above constraints, $4(n-1)$ simultaneous equations are obtained. Solving those equations, the values for $a, b, c, d$ coefficients for every segment are obtained.

Similarly the quintic splines is formulated by joining of piecewise quintic polynomial segments of order 5 . In this case, $6(n-1)$ coefficients are to be determined considering the constraints applicable to this particular case.

\subsection{Trigonometric Splines}

Trigonometric spline function of $m^{\text {th }}$ order varying with time has the following properties [15]

- $y(t)$ is periodic with period $2 \pi$, and is $4 m$ times continuously differentiable.

- $y(t)$ satisfis the interpolation conditions $y_{j}(t)=y_{j}$, $(j=0, \ldots, \mathrm{n})$, where $t_{j}=(0,2 \pi)$.

- In each of the $n$ closed $\operatorname{arcs}\left[\mathrm{t}_{\mathrm{j}}, \mathrm{t}_{\mathrm{j}+1}\right]$, the function $y(t)$ is an element of

Span [1, $\cos (r t), \sin (r t), \operatorname{tcos}(r t), \operatorname{tsin}(r t)]$, $(r=1, \ldots, m)$.

An $\mathrm{m}^{\text {th }}$ order trigonometric spline function $y(t)$ with a total of $2 \mathrm{~m}$ constraints in each of the $\mathrm{n}$ closed $\operatorname{arcs}\left[\mathrm{t}_{\mathrm{i}-1}, \mathrm{t}_{\mathrm{i}}\right]$ $(\mathrm{i}=1, \ldots, \mathrm{n})$ can be represented as

$$
\begin{gathered}
y(t)=y_{i}(t) \quad t=\left[\mathrm{t}_{\mathrm{i}-1}, \mathrm{t}_{\mathrm{i}}\right] \\
y(t)=a_{i, 0}+\sum_{k=1}^{m-1}\left(a_{i, k} \cos k t+b_{i, k} \sin k t\right)+a_{i, m} \cos m\left(t-\gamma_{i}\right) \\
\gamma_{i}=\sum_{j=0}^{2 m-1} \frac{\tau_{i, j}}{2 m}
\end{gathered}
$$

and $\tau_{i, j}$ are the values of $\mathrm{t}$ where $\mathrm{y}_{\mathrm{i}}(\mathrm{t})$ has a constraint applied.
The existence and uniqueness of these functions are guaranteed provided that, for each $i$ and $j, y_{i}^{(r)}\left(\tau_{i, j}\right)$ is not constrained unless $y_{i}^{(r-1)}\left(\tau_{i, j}\right)$ is also constrained $(\mathrm{r}=1,2, \ldots, \mathrm{n})$, where $y^{(r)}$ denotes the $\mathrm{r}$-th order time derivative of $y_{i}$. Here, it can be seen that there are $2 m$ coefficients for each segment of the trigonometric spline, so that $2 m$ constraints on each segment have to be satisfied for finding coefficients. The derivative of the function can be represented as

$$
y^{(r)}\left(t_{i}\right)=y_{i}^{(r)}, \quad r=0, \ldots, m-1, i=0, \ldots, n .
$$

The derivatives at knot points can be chosen by central difference method or they can be chosen by any of the objective function.

In general, each trigonometric polynomial is normalized that is, the spline times $\theta_{i}=t_{i}-t_{i-1}$ are expressed in radians according to the following expression

$$
\theta_{i}=\frac{n \frac{\pi}{m} h_{i}}{T_{\text {tot }}} i=1, \ldots, n
$$

where $h_{\mathrm{i}}$ is the time interval of the $i$-th polynomial (in seconds) and $T_{\text {tot }}=\sum_{i=1}^{n} h_{i}$ is the motion time (in seconds) of the entire trajectory.

If spline intervals (segmental times) are assumed to be equal to each other and the order of the spline fixed as $m=4$, then $\theta_{i}=t_{i}=\pi / 4, \gamma_{i}=\pi / 8, i=1, \ldots, n$.

Once spline interval has been fixed, the spline coefficients can be found easily by multiplying a constant matrix with the vector of the knot angles and derivatives.

The $y^{(r)}\left(t_{i}\right)=y_{i}^{(r)}, r=0, \ldots, m-1, i=0, \ldots, n \quad$ can be chosen by the user or they can be obtained by some optimization procedure like minimization of jerk or overshoots.

\subsection{TEST PROBLEMS AND RESULTS}

\subsection{Single Joint Problem}

In order to analyze the performance of different spline techniques in terms of smoothness, overshoots, and maximum values of velocity and accelerations, a test problem consisting of motion of a single joint was initially formulated.

In this case, one set of joint angles [140, 80, 100, 140, 20] are chosen for the spline interpolations at different time intervals. 

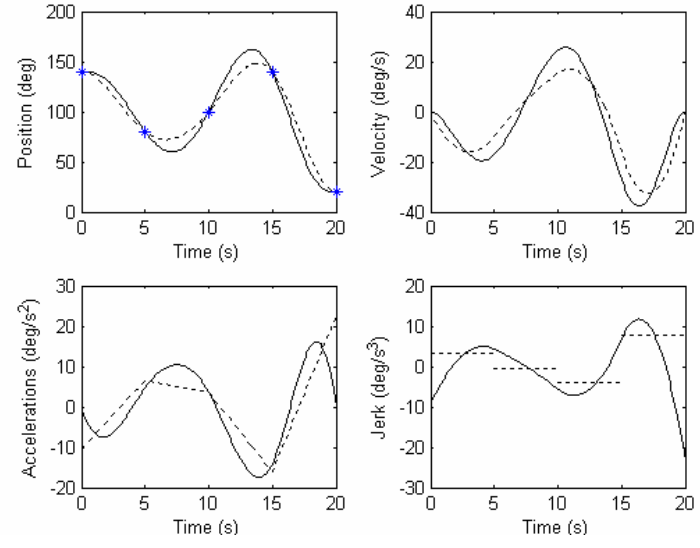

Cubic spline

Qunitic spline

* Knot points

Figure 3. Position, velocity, acceleration, jerk profiles of the $3^{\text {rd }}$ and quintic algebraic splines

The above joint angle set was chosen since the angular displacement in between the knot points covers all values of displacement i.e. minimum, medium, and maximum.
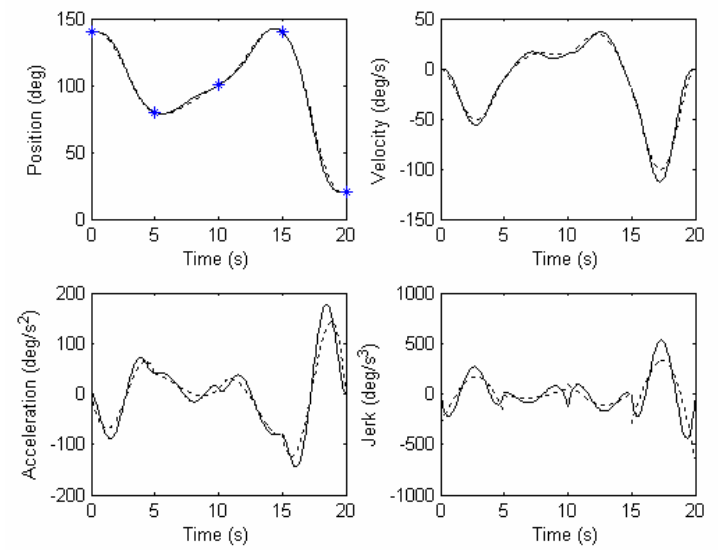

$3^{\text {rd }}$ order Trignometric Spline

$4^{\text {th }}$ order Trignometric Spline

Figure 4 Position, Velocity, Acceleration, Jerk Profiles of the $3^{\text {rd }}$ and $4^{\text {th }}$ order Trigonometric Splines

If two knot points are joined by straight line, the maximum deviation of the trajectory from the straight line is called overshoot. It can also be noticed in Figure 3 that cubic spline has less overshoots and less value of velocities and accelerations when compared to quintic spline. However, the cubic spline only has continuity upto the second order derivative where the quintic spline has continuity upto third order derivative.

Figure 4 shows the comparison of $3^{\text {rd }}$ order and $4^{\text {th }}$ order trigonometric splines for the test problem. It can be observed that there is very little difference between both cases, in terms of overshoots and maximum values of velocities and acceleration. However, the $4^{\text {th }}$ order trigonometric spline has continuity in jerk profile.

Based on the extensive tests on the single joint problem with various spline options, it was found that trigonometric splines outperform algebraic splines in terms of smoothness of path, computational expense, obstacle avoidance, ease of implementation, and optimizability [16]

Further, out of all techniques and options tested, trigonometric $4^{\text {th }}$ order spline with minimizing jerk was found to be appropriate because it has the continuity in jerk profile with minimum values of maximum velocity (maxlvell) and accelerations (maxlaccl). Therefore, this is adapted for cooperative robot trajectory planning.

\subsection{Cooperative Manipulator Problem}

The schematic diagram for cooperative manipulator problem is shown in Figure 5, with robots, object and obstacles position. $R_{1}, R_{2}$ represent the Robot 1 and Robot 2 base position in Cartesian space. 1, 2, 3, 4 represent obstacles and $\mathrm{OB}, \mathrm{OC}$ represent the boom of robot at pick and place positions respectively. It can be observed that the length of $\mathrm{OB}$ and $\mathrm{OC}$ are not equal due to the difference in luff angle and telescope length at pick and place positions.

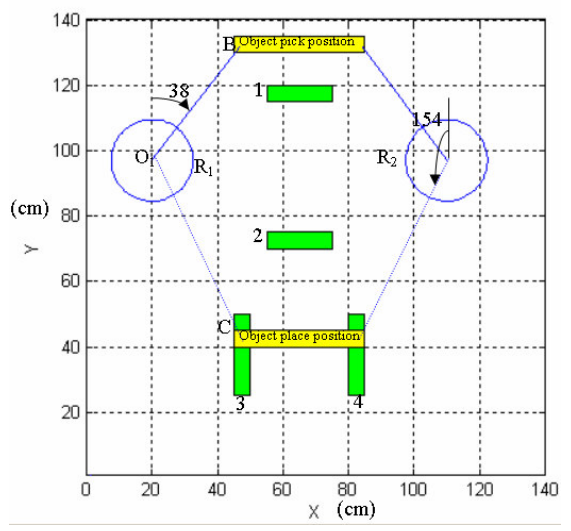

Figure 5. Set-up of Cooperative Manipulator

For this case, the path-points are given by the genetic algorithm. The path-planner considers the obstacles in the workspace, as well as the dimensions of the obstacles and various links [2]. The path is defined in terms successive configurations from pick point to place point and attempts to minimize total work done. In this case, the configurations are limited to 3 points due to the computational complexity in solving the equations.

The three path points used including pick and place points are

$\left\{(38,11,0,4)_{\mathrm{c} 1},(37,11,0,4)_{\mathrm{c} 2},(58,48,12,62)_{\mathrm{c} 1}\right.$, $(62,63,3,62)_{\mathrm{c} 2},(88,46,5,52)_{\mathrm{c} 1},(97,68,6,53)_{\mathrm{c} 2}$, $(137,38,4,50)_{\mathrm{c} 1},(140,51,13,47)_{\mathrm{c} 2},(153,13,15,22)_{\mathrm{c} 1}$, $\left.(154,13,15,22)_{\mathrm{c} 2}\right\}$ 
The trajectories for various joints are generated using the $4^{\text {th }}$ order trigonometric spline with minimizing jerk as objective function. The trajectories of hook points of the two robots in Cartesian space are determined using forward kinematics equations. The corresponding trajectories are shown in Figure 6. For this case, the trajectory profiles of robot 1 and robot 2 are all most same. In more complex cases, the profiles can vary significantly.

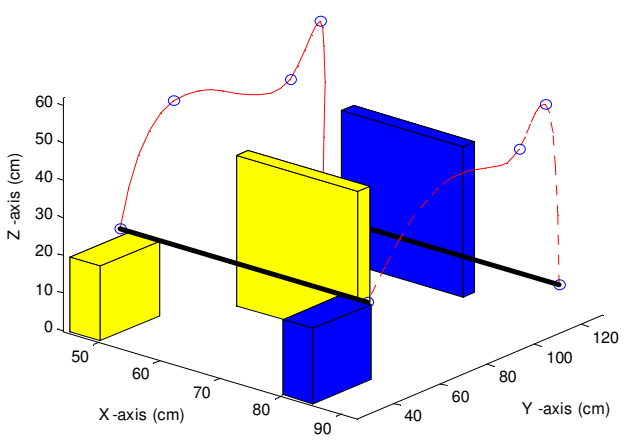

Figure 6. Trajectories of the Hooks of the Two Robots in Cartesian Space

\section{CONCLUSIONS}

Based on this study, it is found that trigonometric splines outperform algebraic splines in the area of smoothness of path, computational expense, obstacle avoidance, ease of implementation, and optimizability. Trigonometric spline of $4^{\text {th }}$ order with minimizing jerk is found to give smooth trajectory with minimal overshoots when compared to other techniques.

The results obtained by applying trigonometric splines for planning the trajectory of cooperative manipulators were feasible and practical. The current problem was simplistic in nature as only 3 path-points were considered. Further work is focused on improving the efficiency of the algorithms, and comparison with scaled manipulator in a construction work-cell.

\section{REFERENCES}

1. Craig, J. J. (1989) Introduction to Robotics, Addison Wesley, Massachusetts.

2. Ajmal Deen Ali, M.S. (2005) Automated motion planning and execution of cooperative manipulators considering obstacles and load interaction aspects, Ph.D Thesis, Department of Mechanical Engineering, Indian Institute of Technology, Madras, Chennai, India.
3. Lin, C.S., Chang, P.R. and Luh, J.Y.S. (1983) Formulation and Optimization of Cubic Polynomial Joint Trajectories for Industrial Robots, IEEE Transactions on Automatic Control, 28(12), 10661074.

4. Simon, D and Isik, C (1991) Optimal trigonometric robot joint trajectories, Robotica, 9, 379-386.

5. Antonio Visioli (2000) Trajectory planning of robot manipulators by using algebraic and trigonometric splines", Robotica, 18, 611-631.

6. Eva Dyllong. and Antonio Visioli (2003), Planning and real time modification of a trajectory using spline techniques, Robotica, 21, 475-482.

7. Isik, C and Simon, D (1993), A trigonometric trajectory generator for robotic arms, Int. J.Control, 57, 505-517.

8. Simon, D. (1997) Neural networks for optimal robot trajectory planning, handbook of neural computation.

9. Magnus Egerstedt, Clyde F. Martin. (2001) Optimal trajectory planning and smoothing splines, Automatics, 37, 1057-1064.

10. Sezimaria Saramago, F.P., Valder Steffen junior (2000) Optimal trajectory planning of robot manipulators in the presence of moving obstacles, Mechanism and Machine theory, 35, 1079- 1094.

11. Lianfang Tian and Curtis Collins (2004) An efficient robot trajectory planning method using a genetic algorithm, Mechatronics, 14, 455-470.

12. Mohri, A., Hirano, G. and Yamamoto, Y. (1996) Cooperative Path Planning for Two Manipulators, Proceedings of the IEEE International Conference on Systems, Man and Cybernetics, 4, 2853-2858.

13.Sun, S., Morris, A.S. and Zalzala, A.M.S. (1996) Trajectory Planning of Multiple Coordinating Robots using Genetic Algorithms, Robotica, 14(1), 227-234.

14. Rana, A.S. and Zalzala, A.M.S. (1996) Near Time Optimal Collision Free Motion Planning of Robotic Manipulators using Evolutionary Algorithms, Robotica, 14, 621-632.

15. Schoenberg, I.J. (1964) On trigonometric spline interpolation, Journal of Mathematics and Mechanics, 13, 795-825

16. Bhaskar S. Vijaya (2005) Collision Free Trajectory Generation For 2x4 Cooperative Robot Using Spline Interpolation And Robot Dynamics, MS Thesis, Department of Mechanical Engineering, Indian Institute of Technology, Madras, Chennai, India. 